\title{
La Norma ISO 21001:2018 - Apéndice normativo y su impacto en estudiantes de educación superior de Lima
}

\section{The ISO 21001: 2018 Standard - Normative annex and its impact on higher education students of Lima}

\author{
Katelinen Mirian Rivera Paipay \\ Universidad César Vallejo, Perú \\ Miguel Ángel Tupac Yupanqui Bustamante \\ Consultor independiente, Perú
}

Autor por correspondencia: katelinen09@gmail.com; miguelangel.tyb@gmail.com

Fecha de recepción: 04 de septiembre del 2019 - Fecha de aceptación: 05 de diciembre del 2019

\section{Resumen}

El propósito de la investigación es determinar el impacto de la norma ISO 21001:2018 - apéndice normativo en estudiantes del último semestre del programa de educación pre escolar de una institución de educación superior de Lima. Es de coyuntura analizar temas de calidad educativa, puesto que los sistemas educativos de diversas partes del mundo, vienen analizando el servicio que están brindando a su público objetivo: estudiantes. Las instituciones que brindan servicios de educación están alineando sus sistemas de gestión de la calidad acogiéndose a normativas que permitan garantizar esa calidad educativa que los visibilice como opciones atractivas. La investigación es de tipo descriptivo y explicativo, el diseño de investigación es no experimental porque solo se hace un análisis de impacto entre las variables. La técnica de investigación ha sido la encuesta. La población está conformada por 150 estudiantes del último semestre del programa de educación pre escolar, teniendo una muestra de 109 estudiantes con un nivel de confianza del $95 \%$ y un margen de error del 5\%. Finalmente se concluye que tiene un impacto positivo en estudiantes del último semestre del programa de educación pre escolar de una institución de educación superior de Lima.

Palabras clave: calidad; impacto; norma ISO 21001; apéndice normativo; estudiantes

\begin{abstract}
The purpose of the research is to determine the impact of ISO 21001: 2018 - normative appendix in students of the last semester of the preschool education program of a higher education institution in Lima. It is a matter of analyzing issues of educational quality, since the educational systems of various parts of the world have been analyzing the service they are providing to their target audience: students. The institutions that provide education services are aligning their quality management systems in accordance with regulations that guarantee that educational quality that makes them visible as attractive options. The research is descriptive and explanatory, the research design is not experimental because only an impact analysis is made between the variables. The research technique has been the survey. The population is made up of 150 students from the last semester of the pre-school education program, with a sample of 109 students with a 95\%
\end{abstract}


confidence level and a 5\% margin of error. Finally, it is concluded that it has a positive impact on students in the last semester of the pre-school education program of a higher education institution in Lima.

Key words: quality; impact; standard ISO 21001; normative appendix; students

\section{Introducción}

A nivel mundial y a lo largo de la historia, la educación, es un tema que siempre está inmerso dentro de las conversaciones de los diferentes estratos sociales como referente de un mañana promisorio y de mejora para las futuras generaciones. Para lograrlo se han definido diversos tipos de políticas educativas, reformas y demás a lo largo del tiempo, y con las mejoras tecnológicas y de la información se han ido instaurando nuevos conceptos que empiezan a regir de manera global; dentro de ellos tenemos uno que ha tomado protagonismo en estos últimos tiempos y está siendo la abanderada de un cambio donde no solo está incluida la formación de estudiantes; sino abarca también diversos temas como son la forma de enseñanza, mecanismos de cómo se imparte la enseñanza, infraestructura, revisiones periódicas de vigencia de contenidos y demás que generan la denominada Calidad Educativa.

Para poder lograr lo anteriormente indicado existen diversos mecanismos pero el que más ha tomado relevancia en estos últimos años en el contexto educativo ha sido el de los sistemas de gestión de calidad (SGC) bajo la normativa de la International Organization for Standarization (ISO) con su norma ISO 9001 que es una norma internacional centrada en todos los elementos de la gestión de la calidad con los que una empresa o institución debe contar para tener un sistema efectivo que le permita administrar y mejorar la calidad de sus productos o servicios. No obstante, viendo a nivel global la necesidad de contar con una normativa propia referida a la educación se establece en el año 2018 la norma ISO 21001:2018, la cual cuentan con 7 anexos de los cuales uno es de tipo Normativo y es motivo del presente artículo.

En este artículo se detallará primero la idea general por la cual parte la ruta de la calidad educativa y cuál es la relación que ahora encuentra en una norma ISO, se comentará de manera general conceptos sobre las normas ISO y su relación con la educación para llegar a abordar también de manera general a la Norma ISO 21001:2018.

En la segunda parte conoceremos de manera genérica el apéndice normativo de la norma ISO 21001:2018 y analizaremos su impacto en estudiantes de educación superior de Lima, observando resultados estadísticos que nos servirán como guía de referencia para establecer si los estudiantes tienen conocimiento sobre este tema importante de actualidad relacionado a sus competencias profesionales.

En la tercera parte se brindarán conclusiones y recomendaciones generadas después de la investigación a manera de poder generar el entusiasmo necesario para que se promuevan posteriores artículos debido a lo novedoso del tema en el plano educativo. 


\section{Problemática}

La agenda al 2030 y los objetivos de desarrollo sostenible refieren dentro de uno de sus objetivos, el número 4, estar orientados a garantiza una educación inclusiva, equitativa y de calidad promoviendo oportunidades de aprendizaje durante toda la vida para todos. El Consejo para la Acreditación de la Educación Superior (CHEA) que es el organismo coordinador más importante de los EE.UU. para la autorregulación de calidad en la educación mediante la acreditación, es otro ejemplo de cómo la calidad educativa esta ya inmersa con el objetivo de asegurar que la enseñanza proporcionada por las instituciones de educación superior cumpla ciertos niveles de calidad académica para la comunidad educativa. En el Perú el Sistema Nacional de Evaluación, Acreditación y Certificación de la Calidad Educativa (SINEACE) es un organismo cuya finalidad es garantizar a la sociedad que las instituciones educativas públicas y privadas ofrezcan un servicio de calidad.

Dentro de este baraje de organizaciones e instituciones referidas al tema de calidad educativa comenzamos a observar que para poder lograr ser acreditas en calidad es necesario una transformación de la escuela tradicional a una escuela activa y abierta que pueda absorber nuevos desafíos y que rompa los paradigmas tradicionales en busca de la excelencia. Es así que para conseguirlo este propósito no se trate de un tema que se logre resolver de la noche a la mañana sino es pasar por todo un proceso, termino importante en la calidad educativa puesto que se tiene que trabajar justamente en eso alinear los procesos de la organización a los objetivos educativos que se buscan, realizando así todo un mapeo, identificación y mejora de los mismos; he aquí un aliado mundial de la famosa estandarización de los procesos, las Normas ISO 9001 que desde 1987 ha estado enfocadas a los sistemas de gestión de calidad, han ido evolucionando y estableciendo el marco de referencia para la gestión de la misma y que han logrado ser adoptadas en un principio por grupos empresariales para determinados sector productivos pero que ha llegado hasta como es el caso, la educación.

Podemos mencionar brevemente la norma ISO 9001-2008 como una de las primigenias adoptadas por algunas instituciones educativas, siendo un modelo algo rígido porque está orientado al producto; al ver este punto ISO dentro de su forma de manejar la mejora continua lanza la versión ISO 9001-2015 que ya tiene una mejor performance hablando en temas educativos puesto que los cambios que contiene están orientados al producto y servicio que en líneas generales puede ser más adaptable a las instituciones educativas, con una denominación de cliente referida al consumidor de los productos y servicios educativos que estas ofrecen.

Como ya se había mencionado teniendo en cuenta que la Educación es un tema muy particular porque no solo se trabajan productos y servicios en las instituciones con miras a una educación de calidad, sino también se tiene mucha interacción con la comunidad académica, ISO volvió a evaluar la connotación de que se necesitaba una norma especial que este dedicada y utilice terminología apropiada para el sector educativo, es entonces que para el año 2018 se presenta oficialmente la Norma ISO 21001:2018 que proporciona una herramienta de gestión común para las empresas educativas con el objetivo de mejorar sus procesos y atender todas las necesidades y expectativas de las personas que utilizan sus servicios. La norma pasa por 10 puntos importantes para ser implantada en las organizaciones educativas y además contiene 7 anexos, del cual uno, el Anexo A tiene el carácter de Normativo y es porque contiene los 
requisitos adicionales para la educación de la primera infancia, o educación pre escolar. Anexo que para el presente trabajo fue clave ya que permitió indagar sobre si los estudiantes de una institución educativa superior de Lima estaban al tanto de estas normativas internacionales que están marcando un momento en la calidad educativa del país y son esenciales en la formación de sus competencias como futuros docentes de dicho nivel educativo.

La norma ISO 21001:2018 como tal, describe un sistema de gestión dirigido a organizaciones educativas de todo tipo, cubre todo el ciclo educativo desde la educación pre escolar hasta los estudios de post grado. Los requisitos de esta norma son genéricos y lo que pretende es que sean aplicables a todas las organizaciones que utilizan un currículo para apoyar el desarrollo de la competencia a través de la enseñanza, el aprendizaje o la investigación sin importar el tipo, tamaño o método educativo de prestación. La norma además se puede aplicar a organizaciones educativas dentro de organizaciones más grandes cuyo negocio principal no es la educación, como los departamentos de formación, capacitación o entrenamiento de tipo académico. La norma no es aplicable a organizaciones que solo producen o fabrican productos educativos.

La norma ISO 21001:2018 como tal también tiene dos enfoques importantes de resaltar, que está orientada a procesos y su pensamiento está basado en riesgos. Entendiendo que estar orientado a procesos en la organización es controlar las interrelaciones e interdependencias entre los procesos del sistema, de modo que se pueda mejorar el desempeño global de la organización, en el caso de la norma ISO 21001:2018 utiliza el ciclo de mejora continua o Planificar-HacerVerificar-Actuar (PHVA). Y el pensamiento basado en riesgos es Abordar tanto los riesgos como las oportunidades estableciendo una base para aumentar la eficacia del sistema de gestión de calidad, alcanzar mejores resultados y prevenir los efectos negativos.

\section{Tabla 1.}

Distribución general de contenidos de la Norma ISO 21001

\begin{tabular}{ll}
\hline Norma ISO 21001:2018 \\
\hline 1. Objeto y campo de aplicación \\
2. Referencia normativas \\
3. Términos y definiciones \\
4. Contexto de la organización \\
5. Liderazgo \\
6. Planificación \\
7. Apoyo \\
8. Operación \\
9. Evaluación del desempeño \\
10. Mejora \\
- Anexo A (normativo) Requisitos adicionales para la educación de la primera infancia. \\
- Anexo B (informativo) Principios para un SGOE. \\
- Anexo C (informativo) Clasificación de partes interesadas en organizaciones educativas. \\
- Anexo D (informativo) Directrices para la comunicación con las partes interesadas \\
- Anexo E (informativo) Procesos, medidas y herramientas en organizaciones educativas. \\
- Anexo F (informativo) Ejemplo de mapeo a estándares regionales. \\
- Anexo G (informativo) Consideraciones de salud y seguridad para organizaciones educativas.
\end{tabular}

Fuente: elaboración propia 


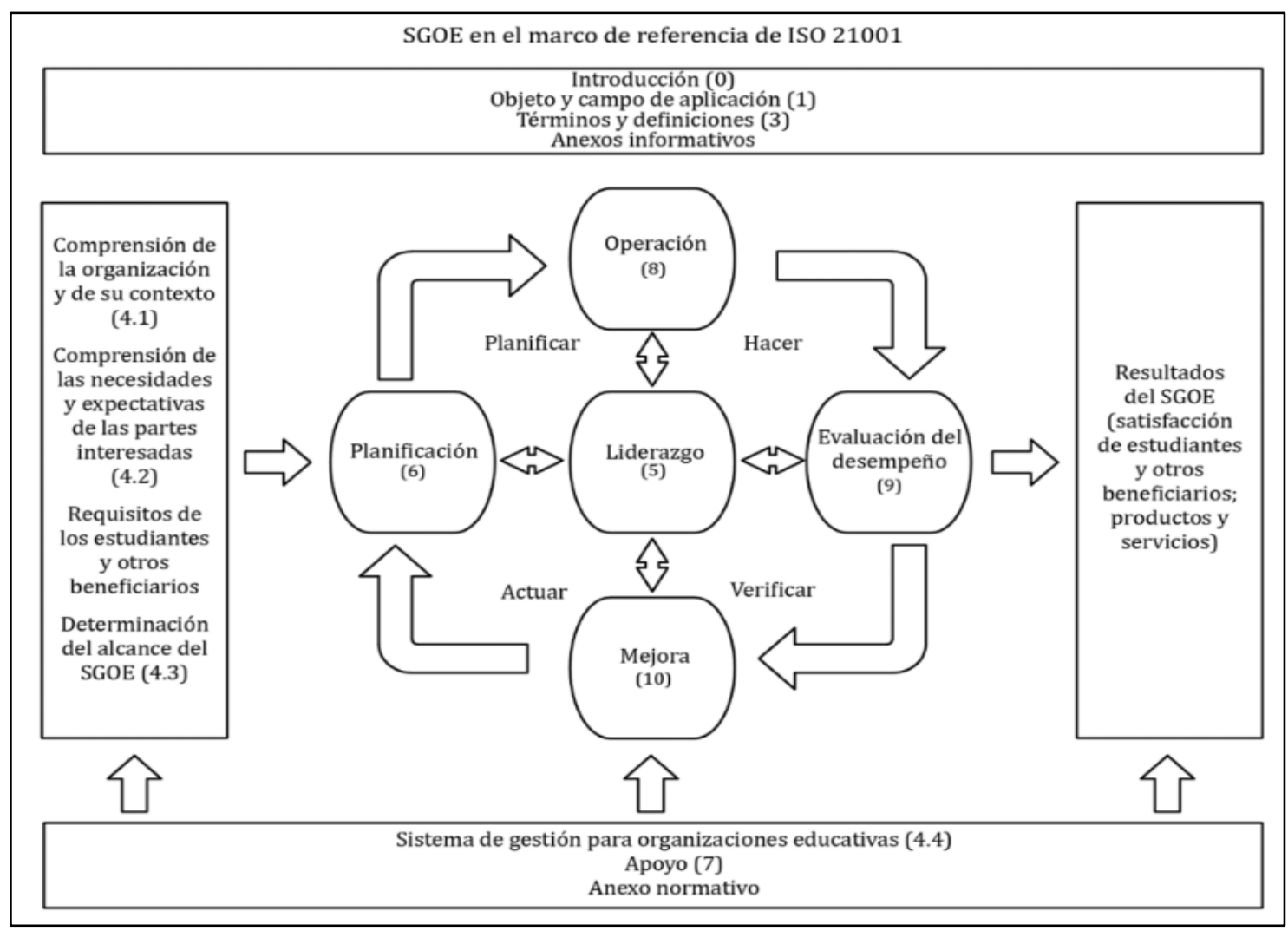

Figura 1. Representación de la estructura del SGC de la Norma ISO 21001:2018 con el ciclo de PHVA.

Después de ver lo anteriormente indicado nos enfocaremos con mayor relevancia en el Apéndice A Normativo que está dirigido a los requisitos adicionales para la educación de la primera infancia, entendiéndose con esto que dicho anexo cumple un rol directriz enfocado a la etapa pre escolar, educación de la primera infancia o también denominada nivel 0 según la clasificación internacional normalizada de la educación (CINE) y que esta descrita como tal en este apéndice.

El Apéndice A Normativo cuenta con 11 puntos relevantes y muy claros enfocados en este nivel educativo que van desde las generalidades, pasando por los principios por los cuales se tiene este apartado especial, el tema de instalaciones que tiene requisitos adicionales y particulares a lo de los demás niveles educativos, competencias que son también especializadas para este nivel educativo, comunicación en las organizaciones de este nivel educativo, sus planes de aprendizaje, otros puntos sensibles a esa edad y nivel educativo como son la recepción y entrega del niño, cuidado de la higiene, cuidado en caso de enfermedad, los materiales, equipos y espacios pedagógicos-lúdicos y la gestión del comportamiento y prevención de la negligencia y el abuso infantil. Como se puede apreciar los puntos descritos son más que suficientes para entender la razón de que este apéndice se haya consignado como normativo pues guarda mucha relación con el trato especializado, dedicado y es de suma importancia pues a ese nivel se tiene la primera relación de los docentes con los niños en las instituciones educativas.

Partiendo de todos estos puntos sobre la Norma ISO 21001:2018 y en especial del apéndice A normativo se procedió a realizar una investigación descriptiva sobre el impacto de este apéndice normativo en estudiantes del último semestre de la carrera profesional de 
educación pre escolar (educación inicial en Perú) de una institución educativa superior de Lima; el cual partió tomando la premisa de la evaluación de impacto de proyecto, la cual valora los resultados y efectos cuantitativos y cualitativos de los proyectos, permitiendo conocer el comportamiento y en este caso el impacto en las condiciones de los futuros profesionales respecto al conocimiento de este anexo normativo.

Para el análisis de este artículo se tomaron dos variables: La primera variable conformada por la "Norma ISO 21001:2018 - Apéndice A" que tomo tres puntos de referencia que fueron la ética y el fomento al desarrollo integral, el ejercicio profesional y el plano organizacional. Y la segunda variable que estuvo formada por las dimensiones cognoscitiva, actitudinal y afectivo emocional tomadas del esquema de dimensiones básicas de toda competencia del libro competencias, calidad y educación superior (2006).

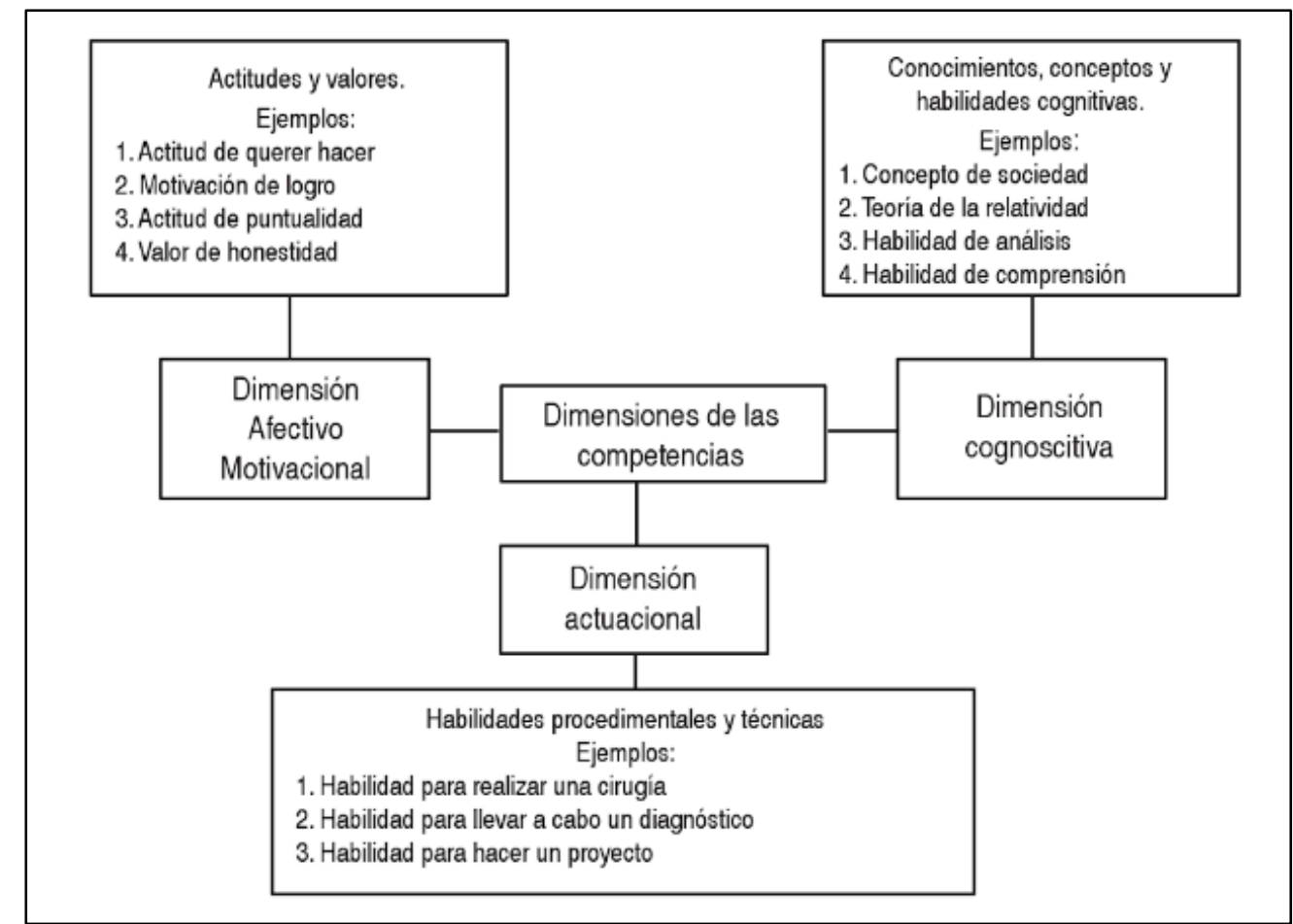

Figura 2. Dimensiones básicas de toda competencia, tomado del libro competencias, calidad y educación superior. (2006)

Dicho esquema fue adaptado a las competencias específicas de la carrera profesional de educación pre escolar de los estudiantes de último semestre de la institución educativa superior de lima teniendo en cuenta lo siguiente: 


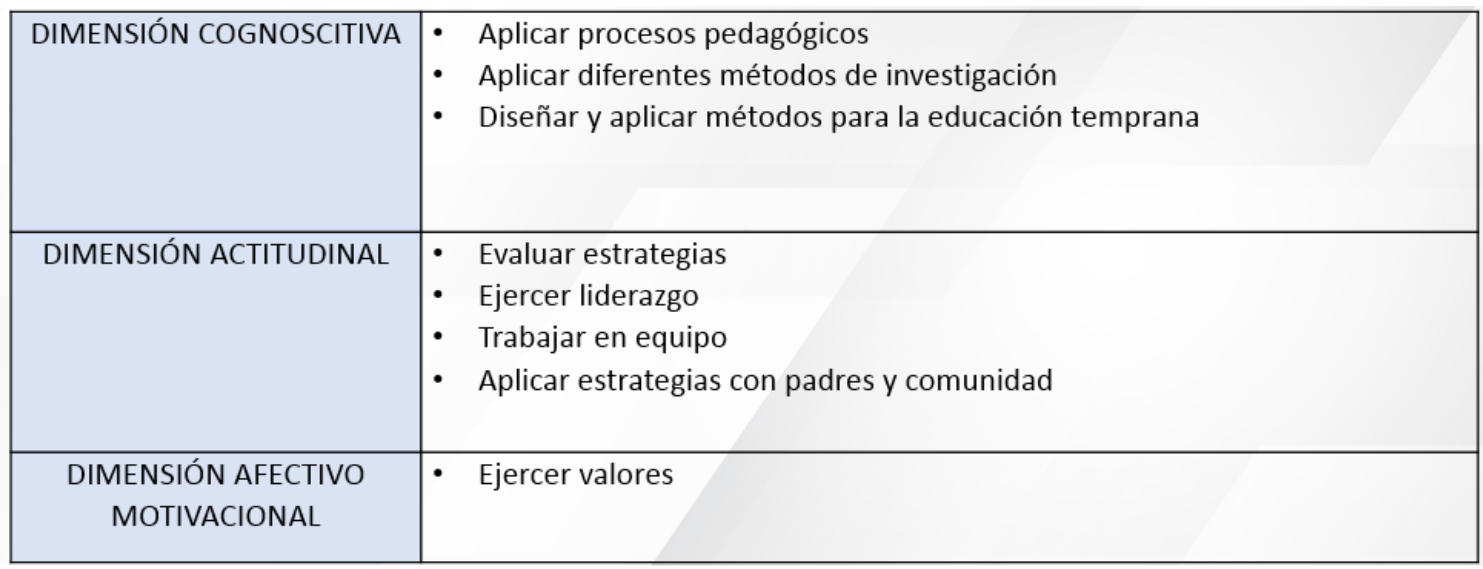

Figura 3. Dimensiones de la variable 2. Adaptado de dimensiones básicas de toda competencia. (2006)

\section{Hipótesis}

Existe relación significativa entre calidad educativa en la primera infancia y el perfil del egreso en los estudiantes de nivel superior de una institución educativa superior de lima.

\section{Método}

La investigación es de nivel descriptivo con el cual se busca especificar las propiedades, las características y los perfiles de personas, grupos, comunidades, procesos, objetos o cualquier otro fenómeno que se someta a un análisis. Es decir, únicamente pretenden medir o recoger información de manera independiente o conjunta sobre los conceptos o las variables a las que se refieren.

Es de tipo correlacional pues tiene como finalidad conocer la relación o grado de asociación que exista entre dos o más conceptos, categorías o variables en una muestra o contexto en particular. En ocasiones sólo se analiza la relación entre dos variables, pero con frecuencia se ubican en el estudio vínculos entre tres, cuatro o más variables.

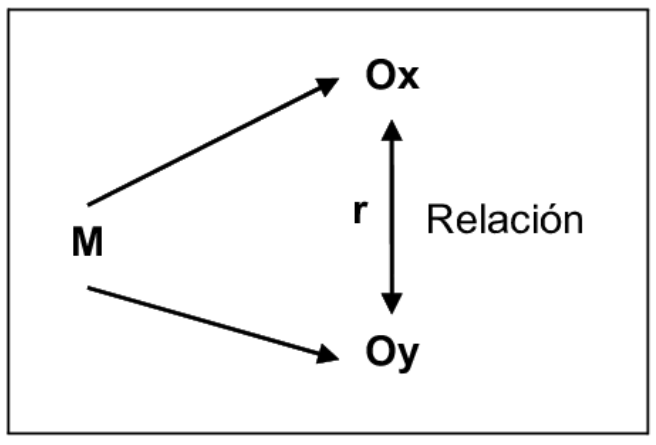

Figura 4. Esquema de una investigación de tipo correlacional.

Donde:

Ox: Variable 1

Oy: Variable 2 
r: Relación

M: Muestra

Es de corte transversal puesto que los datos que se recopilan para estudiar a la población se realizaron en un solo punto en el tiempo con la finalidad de examinar la relación entre variables de interés.

La técnica que se empleó en la siguiente investigación es la encuesta. La cual es un procedimiento dentro de los diseños de investigación descriptiva en el que el investigador recopila datos mediante un cuestionario previamente diseñado, sin modificar el entorno ni el fenómeno donde se recoge la información

El instrumento empleado en el siguiente artículo fue el cuestionario que en fenómenos sociales, tal vez es el instrumento más utilizado para recolectar los datos. Un cuestionario consiste en un conjunto de preguntas respecto de una o más variables a medir.

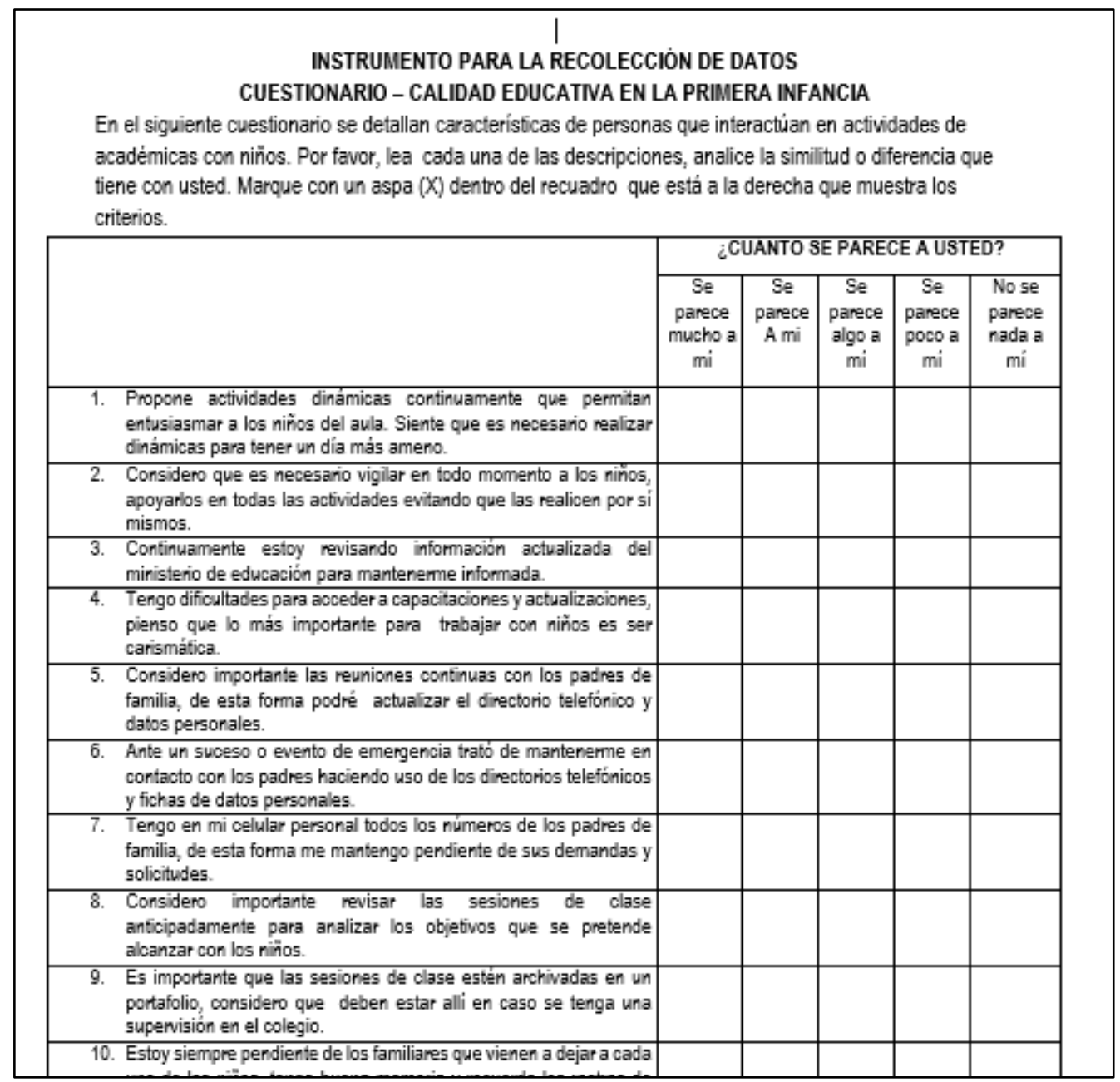

Figura 5. Vista referencial del cuestionario calidad educativa en la primera infancia. 


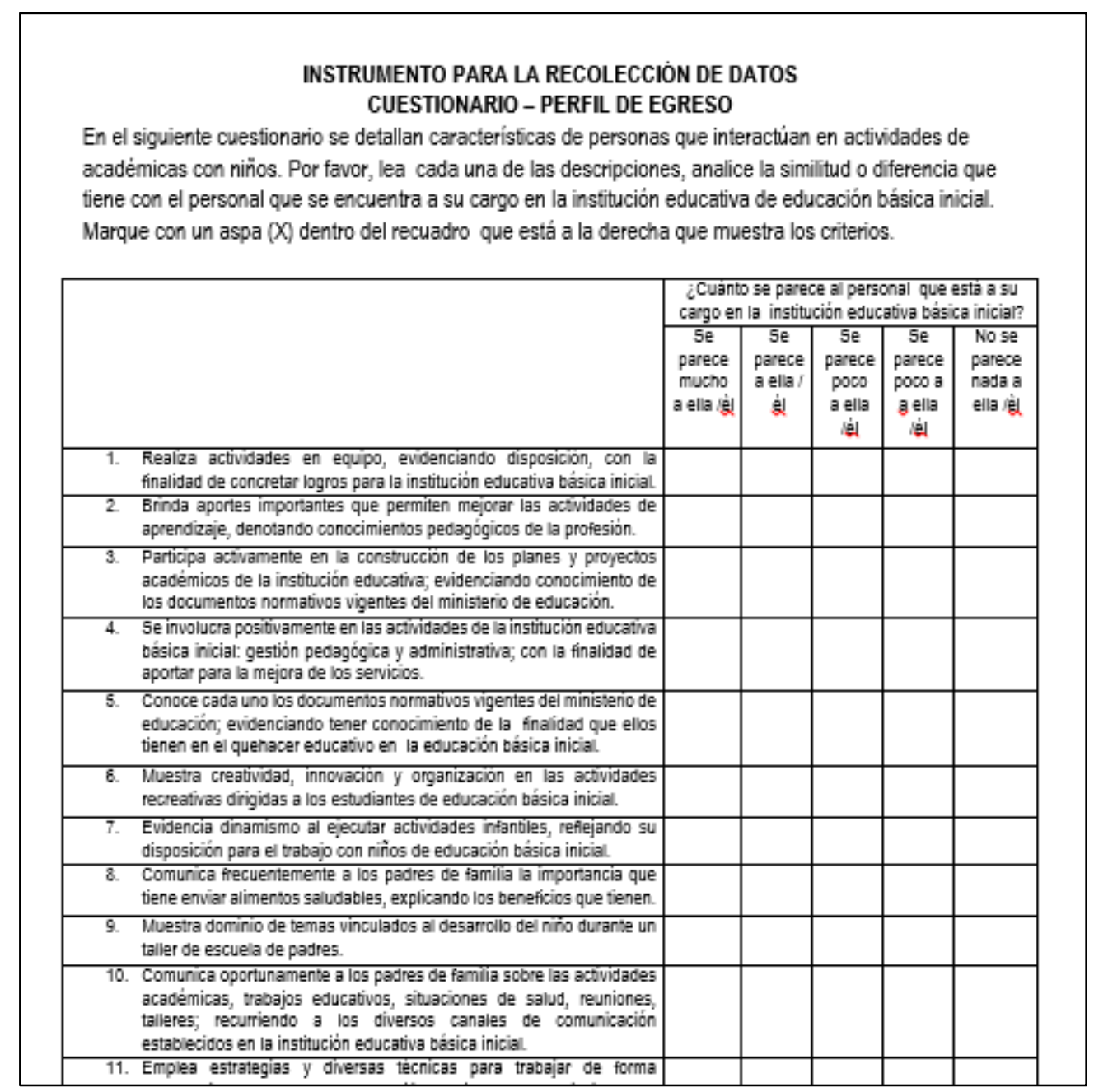

Figura 6. Vista referencial del cuestionario - perfil de egreso.

Cada cuestionario contiene 16 preguntas con alternativas de respuesta para marcar, dichas alternativas están basadas en la escala de Likert y para una mejor apreciación se muestra la distribución de las preguntas basadas en las variables trabajadas en esta investigación y la relación que guardan con las dimensiones de las mismas.

\section{Tabla 2.}

Distribución de preguntas según tipo de cuestionario y dimensiones de variables.

\begin{tabular}{lll}
\hline Cuestionario & Dimensiones & Preguntas \\
\hline Calidad Educativa En La Primera Infancia & Ética y fomento al desarrollo integral & $1 / 2 / 12 / 13 / 15$ \\
& Ejercicio profesional & $3 / 4 / 8 / 9 / 14 / 16$ \\
& Organizacional & $5 / 6 / 7 / 10 / 11$ \\
Perfil De Egreso & & $1 / 4 / 8 / 9 / 10$ \\
& Dimensión actitudinal & $2 / 3 / 5 / 6 / 11 / 13$ \\
& Dimensión cognoscitiva & $7 / 12 / 14 / 15 / 16$ \\
\hline
\end{tabular}

Fuente: elaboración propia 


\section{Método de análisis de la información}

\section{Coeficiente del Alfa de Cronbach}

\section{Tabla 3.}

Confiabilidad de la variable calidad educativa en la primera infancia en los estudiantes de una institución educativa de nivel superior de Lima.

\begin{tabular}{ccc}
\hline Alfa de Cronbach & Alfa de Cronbach basada en elementos estandarizados & N de elementos \\
\hline, 832 &, 825 & 16 \\
\hline
\end{tabular}

Fuente: elaboración propia.

\section{Tabla 4.}

Confiabilidad de la variable perfil de egreso en los estudiantes de una institución educativa de nivel superior de Lima.

\begin{tabular}{ccc} 
Alfa de Cronbach & Alfa de Cronbach basada en elementos estandarizados & N de elementos \\
\hline, 824 &, 819 & 16 \\
\hline
\end{tabular}

Fuente: elaboración propia

\section{Prueba de normalidad}

Se utilizó la prueba de normalidad de Kolmovorov Smirnov (n > 109) para demostrar si la muestra tiene una distribución normal y asimismo determinar el tipo de proceso estadístico a utilizar.

\section{Tabla 5}

Prueba de normalidad de la variable y dimensiones para determinar el uso del proceso estadístico.

\begin{tabular}{lrrr} 
& \multicolumn{3}{c}{ Kolmogorov-Smirnov $^{\mathrm{a}}$} \\
& Estadístico & $\mathrm{gl}$ & Sig. \\
\hline Ética y fomento al desarrollo integral &, 132 & 109 &, 000 \\
Ejercicio profesional &, 182 & 109 &, 000 \\
Organizacional &, 095 & 109 &, 016 \\
Calidad educativa en la primera infancia &, 113 & 109 &, 002 \\
Actitudinal &, 159 & 109 &, 000 \\
Cognoscitiva &, 179 & 109 &, 000 \\
Afectivo motivacional &, 103 & 109 &, 007 \\
Perfil de egreso &, 102 & 109 &, 007 \\
\hline
\end{tabular}

a. Corrección de significación de Lilliefors

Fuente: elaboración propia. 
Mediante la prueba de normalidad se determinó que la investigación es un estudio no paramétrico porque los resultados obtenidos de acuerdo a las variables y dimensiones no cumplen una distribución normal ( $\mathrm{p}<0.05)$, lo que indica estadísticamente la aplicación de un estudio no paramétrico y corresponde utilizar la prueba de correlación de Spearman.

\section{Contrastación de las hipótesis}

\section{a. Prueba de hipótesis general}

Hi. Existe relación significativa entre calidad educativa en la primera infancia y el perfil de egreso en los estudiantes de nivel superior de una Universidad de Lima metropolitana.

Ho. No existe relación significativa entre calidad educativa en la primera infancia y el perfil de egreso en los estudiantes de nivel superior de una Universidad de Lima metropolitana.

\section{b. Regla de decisión}

Sig. > 0.05; Cuando no se rechaza la hipótesis nula.

Sig. < 0.05; Si se rechaza la hipótesis nula.

\section{c. Estadístico}

Correlación de Rho de Spearman (procesador SPSS v 24.0)

\section{Tabla 6}

Correlación de Rho de Spearman entre calidad educativa en la primera infancia y el perfil de egreso en los estudiantes de nivel superior de una Universidad de Lima metropolitana.

\begin{tabular}{|c|c|c|c|c|}
\hline & & & $\begin{array}{c}\text { Calidad educativa } \\
\text { en la primera infancia }\end{array}$ & Perfil de egreso \\
\hline \multirow[t]{6}{*}{ Rho de Spearman } & $\begin{array}{c}\text { Calidad educativa } \\
\text { en la primera infancia }\end{array}$ & $\begin{array}{l}\text { Coeficiente de } \\
\text { correlación }\end{array}$ & 1,000 &, $947^{* *}$ \\
\hline & & Sig. (bilateral) & . & 000 \\
\hline & & $\mathrm{N}$ & 109 & 109 \\
\hline & Perfil de egreso & $\begin{array}{l}\text { Coeficiente de } \\
\text { correlación }\end{array}$ &, $947^{* *}$ & 1,000 \\
\hline & & Sig. (bilateral) &, 000 & \\
\hline & & $\mathrm{N}$ & 109 & 109 \\
\hline
\end{tabular}

De acuerdo a los resultados se evidencia según la prueba de Correlación de Rho de Spearman una relación de $\mathrm{r}=0.947$ (Correlación positiva muy alta) con una significancia de 0.000 menor que el nivel esperado ( $<<0.05$ ), se acepta la hipótesis alterna, se concluye que: Existe relación significativa entre calidad educativa en la primera infancia y el perfil de egreso en los estudiantes de nivel superior de una institución educativa superior de Lima. 


\section{Conclusiones}

La alta dirección debe realizar monitoreo de sus planes de estudio, esto permitirá que se atiendan las demandas y necesidades de la población objetiva a la que se direccionan sus egresados.

Los programas de estudio deben analizar las capacidades de sus perfiles de egreso con la finalidad de fortalecer desde los primeros cursos de especialidad las competencias de las estudiantes, de esta forma se visualizarán logros en los cursos de prácticas pre profesionales.

El programa de estudios debe hacer difusión de sus políticas de calidad educativa, ellos permitirá que la docentes, estudiantes, directivos y comunidad educativa realicen buenas prácticas haciendo un ejercicio idóneo de la profesión.

Las normas internacionales propician que las organizaciones direccionen sus actividades en búsqueda de la calidad, por ello la alta dirección y la comunidad educativa conocerlas e identificar los beneficios que están aportan.

\section{Recomendaciones}

Establecer planes de mejora que permitan fortalecer las capacidades de las egresadas del programa académico, estableciendo acciones de fortalecimiento de capacidades desde los primeros ciclos.

Considerar los resultados vertidos en la presente investigación, como un aporte que permita realizar mejoras en función de la práctica profesional que vienen realizando en los centros de educación básica inicial.

Las organizaciones deben establecer un mecanismo de comunicación que les permita recoger las impresiones de los empresarios o directivos; recurriendo a técnicas e instrumentos que permitan identificar el logro de las competencias de las futuras egresadas.

\section{Bibliografía}

CHEA. (2019). Council for Higher Education Accreditation. Washington DC, USA. Recuperado de https://www.chea.org/about-accreditation

Hernández, R. (1998). ¿Certificación ISO 9000 en educación?. Colección Pedagógica Universitaria. Recuperado de https://www.uv.mx/cpue/coleccion/N_30/Certificaci\%C3\%B3n.htm

ISO 21001: 2018. (2019). Educational organizations -- management systems for educational organizations -- requirements with guidance for use. Recuperado de https://www.iso.org/standard/66266.html

ISO. (2019). International Standarization for Organization. Ginebra, Suiza. Recuperado de https://www.iso.org/about-us.html

ISOTools. (2016). ISO 9001 en los centros educativos. Blog Calidad y Excelencia. Recuperado de https://www.isotools.org/2016/04/19/iso-9001-centros-educativos/ 
ISOTools. (2017). ISO 21001: Un nuevo estándar de sistema de gestión para organizaciones educativas. Blog Calidad y Excelencia. Recuperado de https://www.isotools.org/2017/03/30/iso-21001-nuevo-estandar-sistema-gestionorganizaciones-educativas/

Martín, J., Varela, C., y Coello, A. (2010). Impacto de la implantación de la norma ISO 9001: 2000 en el Archivo General de la Universidad Complutense de Madrid. Revista española de documentación científica, 33(1), 127-143. Recuperado de https://eprints.ucm.es/11040/1/Art\%C3\%ADculo_AGUCM.pdf

Martínez, J., Tobón, S., y Romero, A. (2017). Problemáticas relacionadas con la acreditación de la calidad de la educación superior en América Latina. Innovación educativa 17(73), 79 96. Recuperado de http://www.scielo.org.mx/scielo.php?pid=S166526732017000100079\&script=sci_arttext\&tlng=pt

Norma ISO 21001:2018. (2018). Organizaciones educativas - Sistemas de gestión para organizaciones educativas - Requisitos con orientación para su uso. Ginebra, Suiza.

Quimi, D. (2019). Sistemas de calidad enfocado a las normas ISO 9001 y 21001: caso Facultad de Ciencias Administrativas de la Universidad de Guayaquil. Revista Universidad y Sociedad, 11(1), 279-288. Recuperado de http://scielo.sld.cu/scielo.php?script=sci_arttext\&pid=S2218-36202019000100279

Rojas, W., Capa, L., y Sánchez, M. (2019). Complementariedad del sistema de gestión de la calidad (SGC) de la educación superior ecuatoriana y el SGC ISO 9001. Revista ESPACIOS, 40(02). Recuperado de https://www.revistaespacios.com/a19v40n02/a19v40n02p19.pdf

Sampieri, R., Fernández, C. y Baptista, P. (2014). Metodología de la Investigación, (6 ed.). México D.F.: Editorial MC GRAW HILL.

SINEACE. (2017). Sistema Nacional de Evaluación, Acreditación y Certificación de la Calidad Educativa. Lima, Perú. Recuperado de https://www.sineace.gob.pe/quienes-somos/

SUNEDU. (2016). Condiciones básicas de calidad en la universidad peruana. Lima, Perú. Recuperado de https://www.sunedu.gob.pe/condiciones-basicas-de-calidad-2/

Tobón, S., Rial, A., Carretero, M. y Garcia, J. (2006). Competencias, calidad y educación superior. Bogotá D.C., Colombia. Cooperativa Editorial Magisterio.

Vidal Ledo, M., \& Morales Suárez, I. (2010). Calidad educativa. Educación Médica Superior, 24(2). Recuperado de http://scielo.sld.cu/scielo.php?script=sci_arttext\&pid=s086421412010000200013 